\title{
Effect of insoles with arch support on gait pattern in patients with multiple sclerosis
}

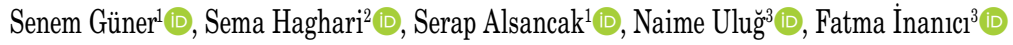 \\ ${ }^{1}$ Department of Prosthetics and Orthotics, Vocational School of Health Services, Ankara University, Ankara, Turkey \\ ${ }^{2}$ Department of Physical Medicine and Rehabilitation, Ethica Hospital, İstanbul,Turkey \\ ${ }^{3}$ Department of Physical Medicine and Rehabilitation, Medicine Faculty of Hacettepe University, Ankara, Turkey
}

Received: November 20, 2017 Accepted: January 22, 2018 Published online: February 25, 2018

\section{ABSTRACT}

Objectives: This study aims to determine the effect of insoles with arch support on gait patterns in patients with multiple sclerosis (MS) and somatosensory impairment.

Patients and methods: Ten patients ( 7 females, 3 males; mean age $34.9 \pm 6.8$ years; range, 48 to 35 years) with clinically definite relapsing remitting MS and age- and sex-matched 10 healthy volunteers (7 females, 3 males; mean age 33.8 \pm 3.2 ; range, 40 to 31 years) were included in the study between January 2011 and January 2012. A medial longitudinal arch and transverse arch supporting polyurethane insole covered with foam shaped using plantar sensory feedback was used. Three-dimensional gait analysis was performed via a Vicon 612 system with six cameras. The participants initially walked barefoot and, then, wore the insoles in their short slipper socks.

Results: All participants were evaluated in terms of kinetics, kinematics, and temporospatial parameters with a gait analysis system. The patients with MS showed improvements in cadence and walking speed when using the insoles. Sagittal plane angles of the hip and knee were increased while using insoles ( $\mathrm{p}<0.05$ ) and ankle plantar flexion was found to be decreased, compared to barefoot walking ( $<<0.05$ ).

Conclusion: Our study results suggest that insole with arch support affects gait cycle, but does not improve gait impairments in patients with MS. Insoles may ensure plantar sensory feedback in feet during walking, which increases pressure in the mid-forefoot area.

Keywords: Gait analysis; insole; multiple sclerosis.

Multiple sclerosis (MS), a demyelinating disease of the central nervous system, is characterized by exacerbations and remissions, affecting movement, cognition, and perception..$^{[1]}$ Gait deficits reduce mobility and independence, leading to falls and injuries and a negative effect on the quality of life of individuals with MS. ${ }^{[2-4]}$ Scheinberg et al..$^{[5]}$ reported $85 \%$ of individuals with MS listed gait disturbances as their main complaint and continued loss of mobility among their greatest concerns for the future. ${ }^{[6]}$ The loss of cutaneous sensation in the plantar surface of the feet has been correlated with impaired balance control and increased risk of falling. ${ }^{[7]}$ Many studies support the important contribution of plantar cutaneous sensation in the control of balance and gait. ${ }^{[8-11]}$ Eils et al. ${ }^{[1]}$ demonstrated that reduced plantar sensation led to significant changes in gait at the ankle, knee, and hip joints, indicating a more cautious pattern of ground contact and push-off with modified electromyographic and motion patterns.

An enhanced sensory feedback from the sole of the foot could combat diminished plantar sensitivity. Sensory feedback from the feet may be influenced by changing the characteristics of a shoe insole or inner surface. Nurse and Nigg ${ }^{[12]}$ investigated the effects of textured footwear on 15 healthy individuals whilst walking with a smooth shoe insert and a textured shoe insert. Reductions in both soleus and tibialis anterior intensity during periods, when these muscles were most active, whilst identified, whilst the participants wore the textured shoe insert. Furthermore, the foot assumed a more plantar-flexed position at heel contact when wearing textured inserts. Kelleher et al. ${ }^{[13]}$ 
demonstrated that, when wearing the textured insoles, there was a significant increase in hip and knee sagittal plane excursion, maximum ankle dorsiflexion, knee flexion and in peak acceleration ground reaction force. Throughout the stance phase, electromyography activity of shank muscles was typically found to increase during wearing the textured insoles.

Current rehabilitation strategies to improve gait and balance in patients with MS have involved various approaches including motor and sensory strategies, robot-assisted gait training, and exercises to address deficit motor function. ${ }^{[14,15]}$ Footwear interventions may be another treatment option to help improve gait performance in MS. ${ }^{[13,16]}$ In the present study, we aimed to determine the effect of insoles with arch supports on gait patterns in a group of patients with MS who experienced plantar sensory deficits.

\section{PATIENTS AND METHODS}

Between January 2011 and January 2012, 10 outpatients with MS (7 females, 3 males; mean age $34.9 \pm 6.8$ years; range, 48 to 35 years) at the rehabilitation clinic at a university hospital were included in this study. Inclusion criteria were as follows: patients with relapsing-remitting MS as diagnosed by a neurologist with a score ranging from 0 to 10 ( 0 , normal neurological examination; those who did not experience a relapse within the past six months, $\geq 6$, limited walking ability and the need to use a technical aid for walking), those with an expanded disability status scale (EDSS) score of between 0 and 6 , and those using MS medications. Exclusion criteria for patients with MS included cognitive disorders, severely impaired visual functions, severe psychiatric disorder or severe arthritis of the knee and hips, pregnancy, any other neurological or vestibular disorders, or high-level spasticity of the lower limbs. Ten age- and sex-matched healthy controls ( 7 females, 3 males; mean age $33.8 \pm 3.2$; range, 40 to 31 years) were also included as the control group. A written informed consent was obtained from each participant. The study protocol was approved by the Medicine Faculty of Hacettepe University Ethics Committee. The study was conducted in accordance with the principles of the Declaration of Helsinki.

\section{Sensation testing}

Light-touch and pressure-sensation thresholds were determined using the Semmes-Weinstein monofilaments. Each filament had a specified diameter and a known buckling force. The 2.83, 3.61, 3.84,
4.17, 4.31, 4.56, 5.07, and 6.65 monofilaments were used in this study. A modified 4, 2, and 1 stepping algorithm was used to evaluate the pressure threshold at three locations on the plantar aspects of both of the participants' feet, including the heel, the medial forefoot and the lateral forefoot. The participants lay on their back in a quiet room and were blindfolded. The monofilaments were applied perpendicular to the skin of the foot being tested for a duration of 1-2 sec. When the examiner announced "now", a stimulus was or was not applied, thus introducing a forcedchoice testing technique. Each test began with the 4.17 filament. Depending on the response of the participant, the changes in stimulus intensity were made in three increments, until the participant's response changed and a turnaround point was reached.

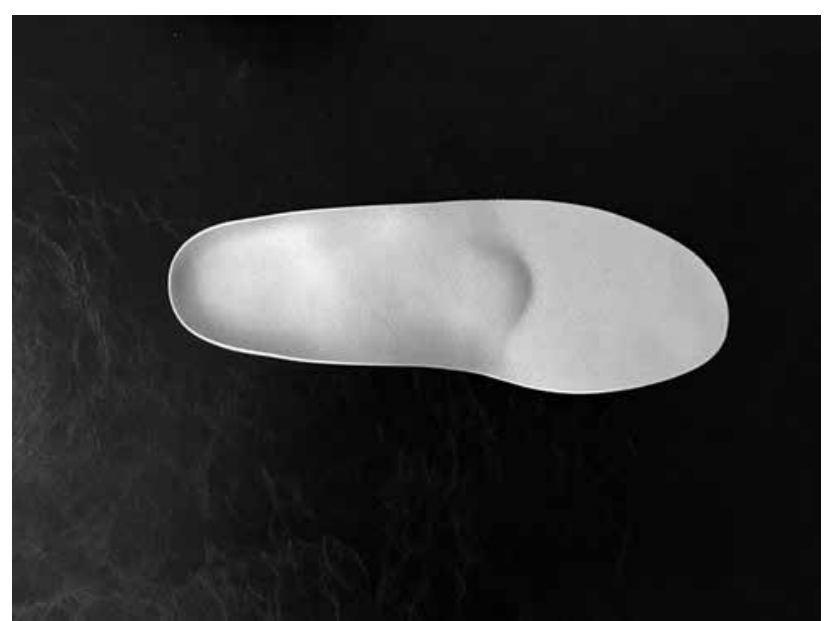

Figure 1. Medial longitudinal arch and transvers arch support insole, superior aspect.

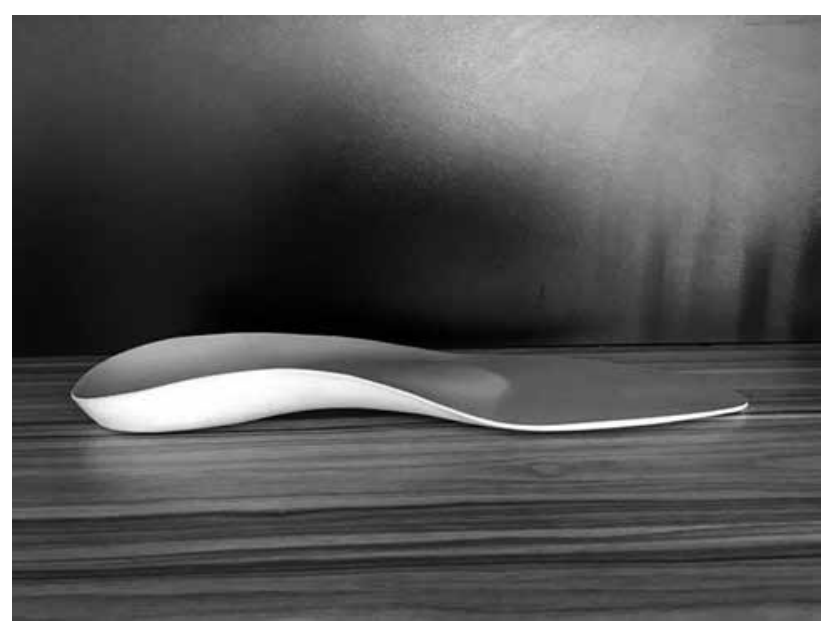

Figure 2. Medial longitudinal arch and transvers arch support insole, lateral aspect. 
Table 1. Descriptive characteristics of study participants

\begin{tabular}{|c|c|c|c|c|}
\hline & \multicolumn{2}{|c|}{ MS patients $(n=10)$} & \multicolumn{2}{|c|}{ Healthy controls $(\mathrm{n}=10$} \\
\hline & $\mathrm{n}$ & Mean \pm SD & $\mathrm{n}$ & Mean \pm SD \\
\hline Age (year) & & $34.9 \pm 6.8$ & & $33.8 \pm 3.27$ \\
\hline Heights $(\mathrm{cm})$ & & $166.7 \pm 10.3$ & & $161.3 \pm 8.0$ \\
\hline Weight (kg) & & $68.8 \pm 13.6$ & & $64.5 \pm 3.0$ \\
\hline \multicolumn{5}{|l|}{ Gender } \\
\hline Male & 3 & & 3 & \\
\hline Female & 7 & & 7 & \\
\hline
\end{tabular}

SD: Standard deviation.

Changes in intensity were subsequently made in twofilament increments, until another turnaround point was reached, at which point all stimuli were presented in one-filament increments. The tactile threshold was determined to be the lightest filament that could be felt $>50 \%$ of the time, with all thicker filaments eliciting a response. For each site tested, the examiner performed a maximum of 25 trials, with five null trials randomly placed throughout the algorithm. If the participant responded to more than two null trials at any given site, the test was halted and the participant was reinstructed.

\section{Insoles}

The insoles were made for both the left and the right feet and were constructed from polyurethane with additional evaluate pyramid ethyl vinyl acetate (EVA) material, forming a medial longitudinal arch and transvers arch. The insoles were covered with $3-\mathrm{mm}$ polyurethane foam and shaped using a grinding and milling machine with a rasp milling tool (Figures 1 and 2). The insole measurements and construction were performed by an orthotic technician.

\section{Gait analysis}

Three-dimensional gait analyses were carried out in the Motion Analysis Laboratory of the Department of Physical and Rehabilitation Medicine at the University Medical School, using the Vicon motion analyses system (Vicon 612 System, Oxford Metrics, Oxford, UK) with six infrared cameras at $50 \mathrm{~Hz}$. The

Table 2. Mean plantar sensation threshold results

\begin{tabular}{|c|c|c|c|c|c|}
\hline & \multicolumn{4}{|c|}{ Plantar sensation } & \multirow[b]{3}{*}{$p$} \\
\hline & \multicolumn{2}{|c|}{ MS patients } & \multicolumn{2}{|c|}{ Healthy controls } & \\
\hline & Median & Min-Max & Median & Min-Max & \\
\hline Medial forefoot & 3.61 & $2.83-4.31$ & 2.83 & $2.44-3.61$ & $0.01^{*}$ \\
\hline Lateral forefoot & 3.61 & $3.61-4.56$ & 3.61 & $2.83-3.61$ & $0.01^{*}$ \\
\hline Heel & 4.08 & $3.61-4.74$ & 3.61 & $2.83-3.61$ & $0.01^{\star}$ \\
\hline
\end{tabular}

Table 3. The result of Wilcoxon signed-rank test in barefoot and with insole spatiotemporal, kinematic and kinetic parameters

\begin{tabular}{|c|c|c|c|c|c|c|c|}
\hline & \multicolumn{2}{|c|}{ MS patients barefoot } & \multicolumn{2}{|c|}{ MS patients wearing insole } & \multicolumn{2}{|c|}{ Healthy controls } & \multirow[b]{2}{*}{$p$} \\
\hline & Median & Min-Max & Median & Min-Max & Median & Min-Max & \\
\hline \multicolumn{8}{|l|}{ Spatiotemporal parameters } \\
\hline Cadence (steps/min) & 103 & $79-125$ & 106.5 & $78.4-132$ & 115.7 & 107-137 & $0.04^{*}$ \\
\hline Double support (sec) & 0.3 & $0.23-0.62$ & 0.28 & $0.19-0.54$ & 0.26 & $0.2-0.34$ & $0.00^{\star *}$ \\
\hline Stride time (sec) & 1.16 & $0.94-1.58$ & 1.12 & $0.91-1.61$ & 1.05 & $0.87-1.14$ & $0.01^{*}$ \\
\hline Walking speed (m/sn) & 1 & $0.5-1.25$ & 1.08 & $0.5-1.25$ & 1.06 & $0.91-1.5$ & $0.002^{*}$ \\
\hline \multicolumn{8}{|l|}{ Kinematic parameters } \\
\hline Hip joint angle at midswing $\left(^{\circ}\right)$ & 31.8 & $18.4-46.9$ & 35.05 & $18.7-41.1$ & 29.1 & $19.5-39.9$ & $0.004^{*}$ \\
\hline Knee joint angle at loading response $\left(^{\circ}\right)$ & 14.5 & $1.88-29.5$ & 15.3 & $0.75-35.8$ & 11.7 & $-2.47-19.29$ & $0.03^{*}$ \\
\hline Knee joint angle at initial swing $\left(^{\circ}\right)$ & 52.7 & $26.5-62.2$ & 56.2 & $18.9-69.2$ & 53.1 & $38.1-57.4$ & $0.006^{*}$ \\
\hline Ankle angle at initial swing $\left(^{\circ}\right)$ & -4.7 & $-9.6-9.9$ & -0.9 & $-10.1-15.79$ & -4.7 & $-16.5-3.5$ & $0.005^{*}$ \\
\hline \multicolumn{8}{|l|}{ Kinetic parameters } \\
\hline Knee power at preswing $(\mathrm{W} / \mathrm{kg})$ & 0.2 & $-2.06-1.36$ & 0.48 & $0.0-0.9$ & -0.79 & $-1.69-0.3$ & $0.02^{*}$ \\
\hline
\end{tabular}


standard plug-in gait marker set (15 markers; one on the sacrum and one each bilaterally on the anterior superior iliac spine, mid-lateral thigh, lateral knee joint, lateral shank, lateral malleolus, on the shoe over the second metatarsal head, and over the posterior calcaneus) was used. Ground reaction forces were measured by two Bertec force plates (Bertec Co., Columbus, OH, USA), which were placed at the middle of a $10-\mathrm{m}$ walkway. Prior to data collection, the camera and force plate were calibrated. The patients were asked to walk at their natural, comfortable speed. Data were collected after several practice trials. For all trials, the participants wore short slipper socks with an insole or were barefoot. Data from five trials for each walking condition were collected and averaged. All data collection was carried out by a single physiotherapist, who was experienced in analyzing gait.

\section{Sample size}

Kelleher et al. ${ }^{[13]}$ found that the mean cadence was $106.73 \pm 7.59$ in healthy individuals. ${ }^{[13]}$ In our study, taking advantage of these values, we would assume that there could be a deviation of \pm 10 in the MS group. The sample size to be taken was calculated as power of $90 \%$ and 0.05 type I error, the minimum sample size to be taken was at least eight individuals.

Statistical analyses were performed using the SPSS version 16.0 (SPSS Inc., Chicago, IL, USA). For comparing changes in gait whilst wearing insoles, compared to without, a non-parametric dependent Wilcoxon signed-rank test was performed. The control group data were used only as reference data to examine whether changes in gait parameters whilst wearing insoles could been construed as positive or negative. No statistical comparisons were performed with this group with the expectation of sensory comparisons with MS group. A $p$ value of $<0.05$ was considered statistically significant.

\section{RESULTS}

Demographic characteristics of the patients and healthy controls are shown in Table 1. Spatiotemporal, kinematic, and kinetic gait parameters were compared between barefoot patients with MS and those with insoles. Statistically significant results are shown in Tables 3, 4, and Figure 3. Baseline spatiotemporal parameters of gait (cadence and walking speed) were reduced, whereas double support and stride time were increased in patients with MS wearing barefoot, compared to those wearing insoles. In patients with MS wearing insoles, the cadence, walking speed, double support, and stride time significantly improved, compared to those with barefoot $(\mathrm{p}<0.05)$. According to kinematic results, hip joint maximum flexion degree increased, when patients were wearing the insoles in the mid-swing phase and knee joint maximum flexion degree increased during the loading response and initial swing phase $(\mathrm{p}=0.006)$. The ankle joint plantar flexion decreased in patients wearing the insoles during the initial swing phase, compared to barefooted patients $(p=0.005)$. Kinetic gait analysis results of power
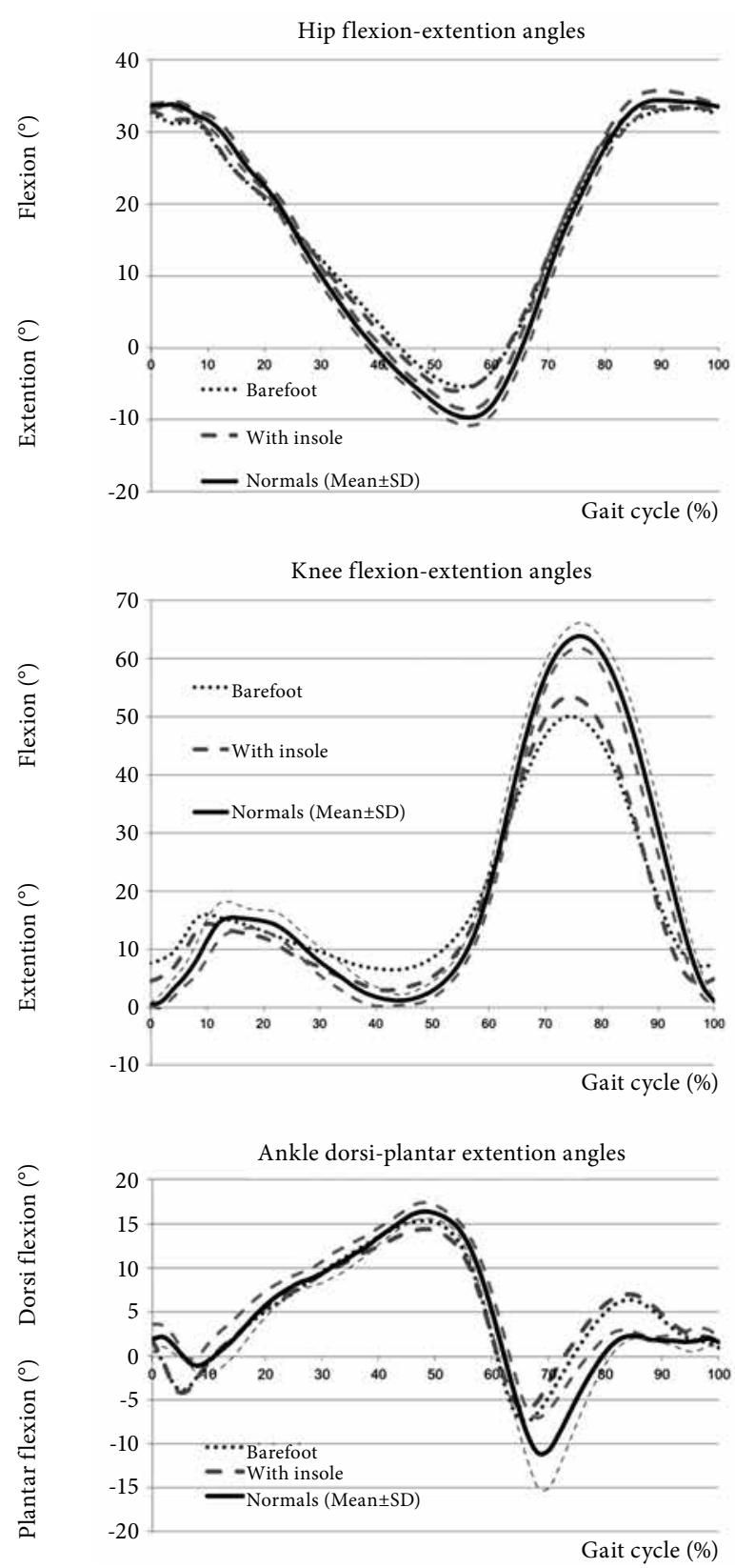

Figure 3. Hip, knee, and ankle joint sagittal plane total excursion degree. 
demonstrated that power knee pre-swing rectus femoris eccentric activity increased, compared to barefooted patients $(\mathrm{p}=0.02)$. The hip and knee joint maximum flexion degree increased during the swing phase, whereas the ankle joint maximum plantar flexion degree decreased during the swing phase. These findings suggested that application of the insoles is particularly effective on the proximal part of the legs. The results of sensation testing at the three sites on the foot in the control group and the MS group exhibited a trend of decreasing sensation with increasing disease severity at all locations (Table 2). For the MS group, the sensation thresholds were highest at the medial forefoot.

\section{DISCUSSION}

The objective of this study was to assess whether applied insole with arch supports affected gait pattern in patient with MS. The patient with MS typically walk slowly with a shorter length and prolonged double support phase, decreased cadence, and lesser joint motion than healthy individuals, which results in reduced community mobility. ${ }^{[17-19]}$ Decreased walking velocity and step length are considered as compensatory for the deficit in balance and postural control. ${ }^{[20]}$ Many studies have reported that textured insoles reduce postural sway during standing in healthy people. $^{[21,22]}$ Our results indicated that insoles had a significant and immediate effect on gait variables, increasing cadence and walking speed. Kelleher et al. ${ }^{[13]}$ reported that textured insoles had several effects on gait kinematics and kinetics, including benefits to knee and hip excursion, and ground reaction forces. According to Kelleher et al., ${ }^{[13]}$ the significant increase in hip and knee excursion could be attributed in some of part to the increase in velocity. Our results also support the findings of Kelleher et al. ${ }^{[13]}$ and we propose that wearing insoles allows the shank segment to generate increased momentum during the swing phase. The results of our study also showed that ankle joint plantar flexion degree decreased and increased hip, knee joint angle during the swing phase with those wearing insole compared to barefoot and healthy groups in the swing phase. Our results suggest that wearing insole may increase plantar sensory feedback; however, the efficacy of insoles remains unclear kinematic parameters in gait cycle. In addition, it is unclear whether insoles with arch support should be recommended for patients with MS. Alfuth $^{[23]}$ reported that the effects textured and other types of stimulating insoles might have been a major reason for the failing. Dixon et al. ${ }^{[16]}$ demonstrated that textured insoles had no significant, immediate effect on gait variables, compared to the control conditions, although they reported an increase in the step and stride length after two weeks of intervention. Kalron et al. ${ }^{[24]}$ did not report any significant changes in spatiotemporal parameters of gait after the fourweek intervention phase. We assume that the different intervention insoles between our study and those of Kalron et al. ${ }^{[24]}$ may have been the cause of this dissimilarity. Kalron et al. ${ }^{[24]}$ used textured insoles that were designed with miniature square pyramids organized in a grid pattern, which did not include arch support. Textured insoles that may change gait are suggested to include the provision of sufficient tactile stimulation to alter the rate of discharge from the mechanoreceptors in feet. Qui et al. ${ }^{[25]}$ reported that wearing shoe insoles increased the stride length and reduced double-limb support time during walking in patients with Parkinson's disease. Similar results have been published in regards to patients with MS, with beneficial alterations in spatiotemporal gait parameters, gait kinetics and kinematics. ${ }^{[13,16]}$

Foot-drop is a common gait problem characterized by a lack of ankle dorsiflexion during the swing phase, which is usually caused by fatigue and muscle weakness. ${ }^{[26]}$ We propose that insoles with medial longitudinal arch and transverse arch supports affect the gait cycle, and ankle joint plantar flexion degree can be reduced with proprioceptive stimulation from under the foot by applying the insole. Other methods of increasing plantar sensory feedback, through techniques such as sandpaper insole, socks with gravel of different size and grade glued to inside of the plantar surface, and textured insoles. ${ }^{[13,21]}$ Ritchie et al. ${ }^{[27]}$ reported that increasing plantar sensory feedback to the medial aspect of the foot reduced the midfoot pronation during walking and, thus, increased the foot positional awareness providing enhanced plantar sensory feedback. Plantar pressure sensation appears to have a particularly important role in controlling balancing reactions that involve rapid compensatory stepping movement. ${ }^{[9-11,28]}$

Plantar foot mechanoreceptors associated with light touch and pressure include the fast adapted (FA) and slow adapted (SA) type I receptors. They form larger receptive fields that are predominantly isolated on the plantar surface of the metatarsaltarsal region of the plantar foot. In the foot, the reflexes are likely to be associated with information related to foot contact (FA I mechanoreceptors) and the provide contact of the foot on a support 
(SA I mechanoreceptors). The activity of mechanoreceptors in the glabrous skin of the foot was shown to cause obvious reflex modulation in ankle muscles. ${ }^{[29,30]}$ According to the results of our study, insoles with added arch supports provided lateral load distribution across the foot and increase the pressure on the mid forefoot area. Thus, insoles affects gait cycle, and reflex arch in the ankle muscle with decreased plantar flexion.

Several limitations of this study should be noted. A major limitation of the present study is that the study group was relatively small. Despite this limitation, wearing insoles was found to be beneficial for improving cadence and walking velocity and but not improved for kinematic parameters in gait cycle patients with MS in this study. A single type of insole was used. It is possible that a different insole and shoes would have produced different effects. In a future study, we would examine the effects of this type of insole on walking, balance, and postural stability in patients with MS and other populations with a higher risk of falling.

In conclusion, insole with arch support is affected gait cycle but not improved gait impairments in patients with MS. Our suggest that wearing insole may increasing plantar sensory feedback but the efficacy of insoles remains unclear kinematic parameters in gait cycle.

\section{Declaration of conflicting interests}

The authors declared no conflicts of interest with respect to the authorship and/or publication of this article.

\section{Funding}

The authors received no financial support for the research and/or authorship of this article.

\section{REFERENCES}

1. Compston A. Non-infective, demyelinating, and paraneoplastic diseases of the nervous system. In: Walton J, editor. Brain's Diseases of the Nervous System. 10th ed. Oxford: Oxford University Press; 1993. p. 366-79.

2. Cameron $\mathrm{MH}$, Lord S. Postural control in multiple sclerosis: implications for fall prevention. Curr Neurol Neurosci Rep 2010;10:407-12.

3. Kalron A, Achiron A. Postural control, falls and fear of falling in people with multiple sclerosis without mobility aids. J Neurol Sci 2013;335:186-90.

4. Pfaffenberger N, Pfeiffer KP, Deibl M, Höfer S, Günther V, Ulmer H. Association of factors influencing health-related quality of life in MS. Acta Neurol Scand 2006;114:102-8.

5. Scheinberg L, Holland N, Larocca N, Laitin P, Bennett A, Hall H. Multiple sclerosis; earning a living. N Y State J Med 1980;80:1395-400.
6. Finlayson M. Concerns about the future among older adults with multiple sclerosis. Am J Occup Ther 2004;58:54-63.

7. Lord SR, Menz HB, Tiedemann A. A physiological profile approach to falls risk assessment and prevention. Phys Ther 2003;83:237-52.

8. Magnusson M, Enbom H, Johansson R, Pyykko I. The importance of somatosensory information from the feet in postural control in man. In: Brandt T, Paulus W, Bles W, Dieterich M, Krafezy S, Straube A, editors. Disorders of Posture and Gait. New York: George Thieme- Verlag; 1990. p. 190-3.

9. Perry SD, McIlroy WE, Maki BE. The role of plantar cutaneous mechanoreceptors in the control of compensatory stepping reactions evoked by unpredictable, multidirectional perturbation. Brain Res 2000;877:401-6.

10. Perry SD, Santos LC, Patla AE. Contribution of vision and cutaneous sensation to the control of centre of mass (COM) during gait termination. Brain Res 2001;913:27-34.

11. Eils E, Behrens S, Mers O, Thorwesten L, Völker K, Rosenbaum D. Reduced plantar sensation causes a cautious walking pattern. Gait Posture 2004;20:54-60.

12. Nurse MA, Nigg BM. The effect of changes in foot sensation on plantar pressure and muscle activity. Clin Biomech (Bristol, Avon) 2001;16:719-27.

13. Kelleher KJ, Spence WD, Solomonidis S, Apatsidis D. The effect of textured insoles on gait patterns of people with multiple sclerosis. Gait Posture 2010;32:67-71.

14. Kasser SL, Jacobs JV, Ford M, Tourville TW. Effects of balance-specific exercises on balance, physical activity and quality of life in adults with multiple sclerosis: a pilot investigation. Disabil Rehabil 2015;37:2238-49.

15. Sangelaji B, Nabavi SM, Estebsari F, Banshi MR, Rashidian $\mathrm{H}$, Jamshidi E, et al. Effect of combination exercise therapy on walking distance, postural balance, fatigue and quality of life in multiple sclerosis patients: a clinical trial study. Iran Red Crescent Med J 2014;16:17173.

16. Dixon J, Hatton AL, Robinson J, Gamesby-Iyayi H, Hodgson $\mathrm{D}$, Rome K, et al. Effect of textured insoles on balance and gait in people with multiple sclerosis: an exploratory trial. Physiotherapy 2014;100:142-9.

17. Crenshaw SJ, Royer TD, Richards JG, Hudson DJ. Gait variability in people with multiple sclerosis. Mult Scler 2006;12:613-9.

18. Gijbels D, Alders G, Van Hoof E, Charlier C, Roelants $\mathrm{M}$, Broekmans $\mathrm{T}$, et al. Predicting habitual walking performance in multiple sclerosis: relevance of capacity and self-report measures. Mult Scler 2010;16:618-26.

19. Givon U, Zeilig G, Achiron A. Gait analysis in multiple sclerosis: characterization of temporal-spatial parameters using GAITRite functional ambulation system. Gait Posture 2009;29:138-42.

20. Syndulko K, Ke D, Ellison GW, Baumhefner RW, Myers LW, Tourtellotte WW. Comparative evaluations of neuroperformance and clinical outcome assessments in chronic progressive multiple sclerosis: I. Reliability, validity and sensitivity to disease progression. Multiple Sclerosis Study Group. Mult Scler 1996;2:142-56.

21. Corbin DM, Hart JM, McKeon PO, Ingersoll CD, Hertel J. The effect of textured insoles on postural control in double and single limb stance. J Sport Rehabil 2007;16:363-72. 
22. Irarrázabal LP, Levy JA, Norr KF, Cianelli R, Issel LM, Pérez $\mathrm{CM}$, et al. Predictors of readiness for oral rapid HIV testing by Chilean health care providers. Rev Panam Salud Publica 2016;40:363-370.

23. Alfuth M. Textured and stimulating insoles for balance and gait impairments in patients with multiple sclerosis and Parkinson's disease: A systematic review and meta-analysis. Gait Posture. 2017;51:132-141.

24. Kalron A, Pasitselsky D, Greenberg-Abrahami M, Achiron A. Do textured insoles affect postural control and spatiotemporal parameters of gait and plantar sensation in people with multiple sclerosis? PM R 2015;7:17-25.

25. Qiu F, Cole MH, Davids KW, Hennig EM, Silburn $\mathrm{PA}$, Netscher $\mathrm{H}$, et al. Effects of textured insoles on balance in people with Parkinson's disease. PLoS One 2013;8:83309.

26. Mount J, Dacko S. Effects of dorsiflexor endurance exercises on foot drop secondary to multiple sclerosis: a pilot study. NeuroRehabilitation 2006;21:43-50.

27. Ritchie C, Paterson K, Bryant AL, Bartold S, Clark RA. The effects of enhanced plantar sensory feedback and foot orthoses on midfoot kinematics and lower leg neuromuscular activation. Gait Posture 2011;33:576-81.

28. Maki BE, Perry SD, Norrie RG, McIlroy WE. Effect of facilitation of sensation from plantar foot-surface boundaries on postural stabilization in young and older adults. J Gerontol A Biol Sci Med Sci 1999;54:281-7.

29. Kennedy PM, Inglis JT. Distribution and behaviour of glabrous cutaneous receptors in the human foot sole. J Physiol 2002;538:995-1002.

30. Fallon JB, Bent LR, McNulty PA, Macefield VG. Evidence for strong synaptic coupling between single tactile afferents from the sole of the foot and motoneurons supplying leg muscles. J Neurophysiol 2005;94:3795-804. 